\title{
On the solution of Stephan inverse problems
}

\author{
Ramin Najafi ${ }^{1}$, Nihan Aliyev ${ }^{2}$ and Ercan Celik ${ }^{1}$ \\ ${ }^{1}$ Ataturk University Faculty of Science, Department of Mathematics, Erzurum-Turkey \\ ${ }^{2}$ Institute of Mathematics and Mechanics of NAS of Azerbaijan, Baku-Azerbaijan
}

Received: 31 December 2015, Accepted: 15 February 2016

Published online: 22 June 2016.

\begin{abstract}
The inverse problem of Stephan in which, beside the unknown function, the much unknown equation, the whole boundary or part of it is unknown for the parabolic equations and recently for hyperbolic equations has been studied.

In this paper the single-phase of Stephan problem will be used taking the Continue method and for every point the answer of the problem will be calculated as definite expressions. It is possible to use this answer as the approximate answer.
\end{abstract}

Keywords: Partial differential equations, Hyperbolic equations, Inverse problem, Stephan inverse problem

\section{Introduction}

In classic differential equation for parabolic equations (heat equation) and hyperbolic equations (string vibration equation), the Cauchy problem and mixed problem, and for the Elliptic equation the boundary value problem of Diriclet and Neumann and special case of Poincare have been studied [1-3].

As we go on, the boundary value problem in elliptic equations the rest of Neuman problem or the Poincare problem in which derivation stamina in the optional boundary (not tangent) is taken into account has been studied [4,5]. After that, to continue those problems the boundary value problem which has the condition of being nonlocal, has been considered in the elliptic equations [6,7].

At first a French mathematician named Hadamard, has shown in the solving of the problems of Mathematical physics that the Cauchy problem is not well-posed for elliptic problems [3,8] and it has been proved later that the boundary value problem is not well-posed for hyperbolic problems, [9] either. Because the mathematical models of the geophysical phenomena have a cauchy problem for elliptic problems. For this reason Tykhonov has found well-posed classes for these problems and later Lavrintov and his students have studied these problems later.

In the problems studied by Tykhonov and Lavrintov sometimes the coefficients of the equation or the coefficients of the boundary conditions and or the right side of the equation is unknown and these problems are called inverse problems. There are some other inverse problems, which are Stephan inverse problems which beside the unknown function of the equation the whole boundary or part of it is unknown. Mostly these problems are applied for the parabolic equations and recently have been applied for the hyperbolic equations too [10-12].

Using the continue method [13] we study the mixed inverse problem for the hyperbolic equations. The advantage of the method compared with the others is that for every point we find the answer analytically as finite expressions. 


\section{The method}

Consider the following problem:

$$
\begin{aligned}
& \frac{\partial^{2} u(x, t)}{\partial t^{2}}=\frac{\partial^{2} u(x, t)}{\partial x^{2}} \\
& \begin{cases}u(x, 0)=\phi(x) & 0<x<\gamma(t), \quad t>0 \\
\left.\frac{\partial u(x, t)}{\partial t}\right|_{t=0}=0 & 0 \leq x \leq \gamma(0)=1\end{cases} \\
& u(0, t)=u(\gamma(t), t)=0, \quad t \geq 0
\end{aligned}
$$

In which $\phi(x)$ and $\gamma(t)$ arbitrary functions have been given and $\phi(0)=\phi(1)=0$ We can easily see that whenever

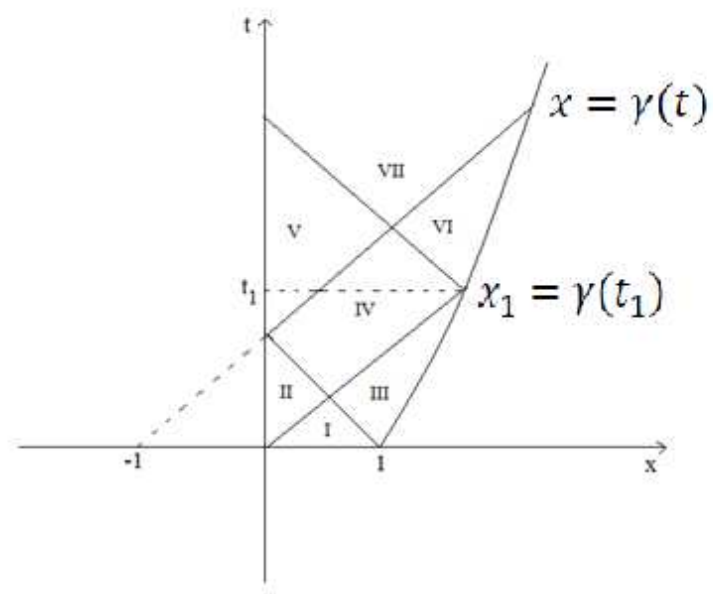

$(x, t) \in I \mathrm{~K}$, the solution to the problem (1)-(3) is not dependent on boundary and boundary condition (3) and the solution is as following:

$$
u(x, t)=\frac{\phi(x-t)+\phi(x+t)}{2}, \quad(x, t) \in I
$$

Whenever $(x, t) \in I I$ characteristic of equation (1) crossing this point, axis $x$ from right in $(0,1)$ and from left in $(-1,0)$. In this case it is necessary that function $(2)$ is extended to $(-1,0)$. For this purpose we have from first boundary condition:

$$
\phi(-x)=-\phi(x)
$$

This means that $\phi(x)$ which has been given in [0,1] interval is continued to left from zero point in the from of odd function, if we consider $\phi_{2}(x)$ as following:

$$
\phi_{2}(x)=\left\{\begin{array}{l}
\phi(x), \quad x \in[0,1] \\
-\phi(-x), \quad x \in[-1,0]
\end{array}\right.
$$

The solution to this mixed problem using this function is as following:

$$
u(x, t)=\frac{\phi_{2}(x-t)+\phi_{2}(x+t)}{2}, \quad(x, t) \in I I
$$


If $(x, t) \in I I I$, characteristic of equation (1) passing this point cut off axis $x$ in $\left(0,2 x_{1}\right)$ interval. For this reason function $\phi(x)$ which is known in $(0,1)$ interval is continued to $\left[1,2 x_{1}\right]$ interval. For this continuation we have from second boundary condition of (3):

$$
\phi(\gamma(t)+t)=-\phi(\gamma(t)-t)
$$

Here $\gamma(t)-t \in[0,1], t \in\left[0, t_{1}\right]$.

We can easily see that $\gamma(0)-0=1, \gamma\left(t_{1}\right)-t_{1}=\gamma\left(t_{1}\right)-x_{1}=0$ In this way if $t \in\left[0, t_{1}\right], \phi(x)$ from [0,1] interval from relation (7) is continued to $\left[1, \gamma\left(t_{1}\right)+t_{1}\right]$ interval.

$$
\gamma\left(t_{1}\right)+t_{1}=x_{1}+t_{1}=2 x_{1}
$$

Thus with definition:

$$
\phi_{3}(x)=\left\{\begin{array}{l}
\phi(x), \quad x \in[0,1] \\
-\phi,(\gamma(x+\sigma(x))-(x+\sigma(x))) \quad x \in\left[1,2 x_{1}\right]
\end{array}\right.
$$

The solution to problem (1)-(3) is:

$$
u(x, t)=\frac{\phi_{3}(x-t)+\phi_{3}(x+t)}{2}, \quad(x, t) \in I I I
$$

Here $t=x+\sigma(x)$ is a solution $\gamma(t)+t=x$ :

$$
\begin{array}{cc}
\gamma(x+\sigma(x))+\sigma(x) & =0 \quad x \in\left[1,2 x_{1}\right] \\
\sigma(t+\gamma(t))+\gamma(t) & =0 \quad t \in\left[1, t_{1}\right]
\end{array}
$$

Point 1: In recent case if we consider function $\phi_{2}(x)$ instead of function $\phi(x)$, we will have the continuation of function in biggest right interval in which the solution to problem is in both III and VI and IV.

We can continue this to left or right. In this case we will have the following theorem:

Theorem 1. Whenever $x \in[0,1] ; \phi(x)$ and $\gamma(x), t \geq 0$ are known function and twice differentiable and $\phi(0)=\phi(1)=0$ and $\gamma(0)=1$ and all the functions and the result of extension of are function in the space of $\mathbb{C}^{2}$ and $\gamma(t)+t=x$ is the unique solution to $t=x+\sigma(x)$, in this case the unique solution to problem (1)-(3) is through step by step method.

Now we deal with Stephans inverse problem. We assume that for $u(x, t)$ functions, $\gamma(t)$ is a unknown function. In this case we add the following condition (1)-(3) problem:

$$
\gamma^{\prime}(t)=\left.\frac{\partial u(x, t)}{\partial(x)}\right|_{x=\gamma(t)}, \quad t \geq 0
$$

In the inverse problem (10) and (1)-(3) like previous mixed problem, if $(x, t) \in I$, the solution to inverse problem (10) and (1)-(3) will be as relation (4). Whenever $(x, t) \in I I$ like previous mixed problem, The solution to inverse problem will be like (6) which has been given using $\phi_{2}(x)$ function in (5). Whenever $(x, t) \in I I I$, because $\gamma(t)$ is an unknown function. We will deal with all possible cases to $\gamma(t)$ :

State 1: $\gamma^{\prime}(t)<-1$, it means that the boundary for small $t$, are placed in $I$ area. Since characteristic of each point in $I$ cut off $x$ axis in [0,1] interval, whenever solutions (1)-(2) which is in the from of (4) are placed in the second condition of (3). We will have:

$$
\phi(\gamma(t)-t)+\phi(\gamma(t)+t)=0, \quad \gamma(t)-t \in(0,1), \quad \gamma(t)+t \in(0,1)
$$


Thus function $\phi(x)$ in [0,1] interval is not arbitrary. Even amounts of this function have different signs for nearly points. This contrasts with the continuity of $\phi(x)$ function. Thus $\phi(x) \equiv 0 ; x \in[0,1]$ as a result $u(x, t)=0$.

State 2: $\gamma^{\prime}(t)=1$; in this case $\gamma(t)$ boundary coincides with $I$ area boundary passing from $x=1$, since this boundary is one of characteristics of equation (1) $\gamma(t)=1-t$ or $\gamma(t)+t=1$ like state 1 we have:

$$
\phi(\gamma(t)-t)=\phi(1-2 t)=-\phi(\gamma(t)+t)=\phi(1)=0, \quad 1-2 t \in[0,1]
$$

In this state the solution to mixed problem will be as $u(x, t)=0$.

State3: $-1<\gamma^{\prime}(t)<0$; in this case $-t<\gamma(t)-\gamma(0)<0$ or $-t<\gamma(t)-1<0$ or $1-t<\gamma(t)<1$

We will have: $1-2 t<\gamma(t)-t<1,1<\gamma(t)+t<1+t$ whenever $\gamma(t)-t \in[0,1]$ then $\gamma(t)+t>1$. This means that from second condition of (3) function $\phi(x)$ is expanded to right from [0,1] area. Because of expansion of function $\phi(x)$ to left, we can consider $\gamma(t)-t \in[-1,1]$, in this case the solution to the equation $\gamma(t)-t=1$ is $t_{1}$, we will have the continuation of function $\phi(x)$ in $\left[1,2 x_{1}\right]$ which $2 x_{1}=\gamma\left(t_{1}\right)+t_{1}$, in this case first we specify function $\gamma(t)$. If we put solution (6) in condition (10), we will have:

$$
\gamma^{\prime}(t)=\frac{1}{2}\left[\phi_{2}^{\prime}(\gamma(t)-t)+\phi_{2}^{\prime}(\gamma(t)+t)\right]
$$

How we put (6) in second condition of (3)

$$
\begin{gathered}
\phi_{2}(\gamma(t)-t)+\phi_{2}(\gamma(t)+t)=0 \\
\phi_{2}^{\prime}(\gamma(t)-t)\left(\gamma^{\prime}(t)-1\right)+\phi_{2}^{\prime}(\gamma(t)+t)\left(\gamma^{\prime}(t)+1\right)=0
\end{gathered}
$$

From this we determine $\phi_{2}^{\prime}(\gamma(t)+t)$ and put in (11), Thus we have:

$$
\gamma^{\prime}(t)=\frac{1}{2}\left[\phi_{2}^{\prime}(\gamma(t)-t)+\frac{1-\gamma^{\prime}(t)}{1+\gamma^{\prime}(t)} \phi_{2}^{\prime}(\gamma(t)-t)\right]
$$

or

$$
\begin{gathered}
\gamma^{\prime}(t)\left(1+\gamma^{\prime}(t)\right)=\phi_{2}^{\prime}(\gamma(t)-t) \\
\left(\gamma^{\prime}(t)\right)^{2}+\gamma^{\prime}(t)-\phi_{2}^{\prime}(\gamma(t)-t)=0
\end{gathered}
$$

The roots of this quadratic in relation to $\gamma^{\prime}(t)$ are:

$$
\gamma^{\prime}(t)=\frac{-1 \mp \sqrt{1+4 \phi_{2}^{\prime}(\gamma(t)-t)}}{2}
$$

We have from(12):

$$
\phi_{2}^{\prime}(\gamma(t)-t)<0
$$

If we want the roots of equation (14) to be real, we should have:

$$
1+4 \phi_{2}^{\prime}(\gamma(t)-t) \geq 0 \quad \text { or } \quad-\frac{1}{4} \leq \phi_{2}^{\prime}(\gamma(t)-t)<0, \quad t \in\left[0, t_{1}\right]
$$


In this case both relations (14) are possible. Thus we deal with both states separately whenever

$$
\gamma^{\prime}(t)=\frac{-1+\sqrt{1+4 \phi_{2}^{\prime}(\gamma(t)-t)}}{2}
$$

In this case we will have for $\gamma(t)$ :

$$
\gamma(t)=1-\frac{t}{2}+\frac{1}{2} \int_{0}^{t} \sqrt{1+4 \phi_{2}^{\prime}(\gamma(\tau)-\tau)} d \tau \quad t \in\left[0, t_{1}\right]
$$

After having function $\gamma(t)$ from (17), we will have the solution to the problem at $(x, t)$ like previous mixed problem. Whenever

$$
\gamma^{\prime}(t)=\frac{-1-\sqrt{1+4 \phi_{2}^{\prime}(\gamma(t)-t)}}{2}
$$

Then we have following equation for $\gamma(t)$

$$
\gamma(t)=1-\frac{t}{2}-\frac{1}{2} \int_{0}^{t} \sqrt{1+4 \phi_{2}^{\prime}(\gamma(\tau)-\tau)} d \tau \quad t \in\left[0, t_{1}\right]
$$

Point 2: In these two cases, since none of relations for $\gamma(t)$ are not in contrast with each other we will have two solutions for $u(x, t)$ and this is not possible because of unique solution of the problem.

State 4: $\gamma^{\prime}(t)=0$; in this case $\gamma(t)=\gamma(0)=1$. In this case we will continue function $\phi(x)$ to right from $x=1$ in the method that we continued this function to left from $x=0$ oddly. This state has already been reviewed $[1,3,8,13]$.

State 5: $0<\gamma^{\prime}(t)<1$ in this state $1<\gamma(t)<1+t$ therefore

$$
1-t<\gamma(t)-t<1 . \quad 1+t<\gamma(t)+t<1+2 t
$$

Considering the expansion of $\phi(x)$ to left we can have: $\gamma(t)-t \in[-1,1]$ in this state the function $\gamma^{\prime}(t)$ has been given as relation (14). From relation (12) we will $\phi_{2}^{\prime}(\gamma(t)-t)>0$. Thus both roots of relation (14) will be real but whenever

$$
\gamma^{\prime}(t)=\frac{-1-\sqrt{1+4 p h i_{2}^{\prime}(\gamma(t)-t)}}{2}
$$

Because of $0<\gamma^{\prime}(t)<1$ we will have $\sqrt{1+4 p h i_{2}^{\prime}(\gamma(t)-t)}<-1$ and this is not acceptable thus we always have in state 5 .

$$
\gamma^{\prime}(t)=\frac{-1+\sqrt{1+4 p h i_{2}^{\prime}(\gamma(t)-t)}}{2}
$$

Therefore whenever we show the solution to $\gamma(t)-t=-1$ with $t_{1}$, we will have the expansion of $\phi(x)$ in $\left(1,2 x_{1}\right]$ area which $2 x_{1}=\gamma\left(t_{1}\right)+t_{1}$. Having $\gamma^{\prime}(t)>0$, thus $\gamma(t)$ is ascending and as a result $t_{1}$ is unique. Considering relation (20) we always have $t_{1}>2$. So we have $\gamma(t)$ :

$$
\gamma(t)=1-\frac{t}{2}+\frac{1}{2} \int_{0}^{t} \sqrt{1+4 \phi_{2}^{\prime}(\gamma(\tau)-\tau)} d \tau \quad t \in\left[0, t_{1}\right]
$$

After getting function $\gamma(t)$ from equation (21), we find the solution to the problem in $(x, t)$ point like previous mixed problem. 
State 6: $\gamma^{\prime}(t)=1$; in this case $\gamma(t)=1+t$ or $\gamma(t)-t=1$ and like state 2 we have

$$
\phi(\gamma(t)+t)=\phi(1+2 t)=-\phi(\gamma(t)-t)=-\phi(1)=0, \quad \gamma(t)+t>1
$$

Then function $\phi$ is as following:

$$
\phi(x)=\left\{\begin{array}{l}
\phi(x), \quad x \in[0,1] \\
-\phi(-x) \quad x \in[-1,0] \\
0 \quad \text { otherwise }
\end{array}\right.
$$

and the solution to $u(x, t)$ is gained from relation (4) with the help of $\phi$ considering $x \in R$

State 7: $\gamma^{\prime}(t)>1$; in this case both characteristices passing from $(x, t)$ cut off $x$ axis on two points out of interval [0,1] and on its right direction. Thus $\phi$ in this area is independent from interval $[0,1]$ therefore considering $\phi(x) \equiv 0 ; x>1$ and considering first condition from (3) we have: $\phi(x) \equiv 0 ; x<-1$ and this state is like state 6 meaning $x \in[-1,1] ; \phi(x) \neq 0$; and $x \notin[-1,1] ; \phi(x)=0$.

\section{Conclusion}

In this paper, we considered a Stephan inverse problem by finding unknown boundary value andobtained it as integral equation. So, this method can be easily to similar inverse problem.

\section{References}

[1] R . Courant and D . Hilbert, Hilbert, Methods of mathematical physics ; Interscience publishers ; volume II,(1962)

[2] V.S.Vladimirov, Equations of mathematical physics ; Mir publishers, Moscow,,(1984)

[3] A.N.Tikhonov and A.A.Samarskii, Equations of mathematical physics ; Pergamon press ,Oxford, (1968)

[4] A.V.Bitsadze, value problems for second-order elliptic equation,North-Hollond,(1968)

[5] T. Y.V.Egorov and V.A.Condrat'ev, ”Mat.Sbor”, 78,148-179,(1969)

[6] N.Aliev and M.Jahanshahi, Sufficent conditions for Reduction of the BVP including a mixed P.D.E with nonlinear boundary conditions to fredholm integral equation, Int.J.Math.Educ.Tech.Scie,28(3),(1997).

[7] G.Kavei and N.Aliev, An analytical method to the solution of the time dependent schrodinger equation using half Cylinder space system-I,Bulletin of pure and Applied sciences 16E(2),253-263,(1997)

[8] I.G.Petrovski, on partial differential equations, Wiley-Interscience New york,(1955).

[9] S.G.Mikhlin, Mathematical physics, an advanced course, North-Holland Amsterdam ,(1970)

[10] A.Fridman and Hub, The Stefan problem for a hyperbolic heat equation, J.Math.Anal.Appl ,138,249-279,(1989).

[11] R.E.Showalter and N.J.Walkington, A hyperbolic stefan problem, Quarter.Appl.Math ,XLV,769-781,(1987).

[12] ] L.M.Desocio and G.Gualtieri, A hyperbolic stefan problem, Quarter.Appl.Math,253-259,(1983).

[13] ] N.S.Koshlyakov, E.B.Gliner and M.M.Smirnov, Partial differential equation of mathematical physics, North-Holland Amsterdam,(1964). 